\title{
Adult urinary bladder tumors with rabdomyosarcomatous differentiation: Clinical, pathological and immunohistochemical studies
}

\author{
Zhanyong Bing ${ }^{*}$ and Paul J Zhang
}

\begin{abstract}
Adult rhabdomyosarcoma (RMS) in the urinary bladder is rare, and is the subject of case reports and small series. It consists of sheets of small round blue cells with high nuclear cytoplasmic ratio, brisk mitosis and apoptosis. In this study, we reported one case of pure rhabdomyosarcoma and two cases of urothelial carcinomas with extensive rhabdomyosarcomatous differentiation. In addition, their immunohistochemical profile was compared to that of small cell carcinoma of the bladder. Our study showed that sufficient sampling was critical for the diagnosis of urothelial carcinoma with extensive rhabdomyosarcomatous differentiation. As adult RMS in the bladder and urothelial carcinoma with rhabdomyosarcomatous differentiation shared morphological features with small cell carcinoma of the bladder, appropriate immunohistochemical stains were necessary in the differential diagnosis. We showed both rhabdomyosarcoma and rhabdomyosarcomatous areas of the urothelial carcinoma were positive for myogenin, negative for cytokeratin and chromogranin stains. In contrast, small cell carcinoma was positive for cytokeratin, and 7 out of 9 cases were also positive for chromogranin. Both rhabdomyosarcoma and small cell carcinoma could be positive for synaptophysin, a potential pitfall to avoid. In addition, all of the tumors with rhabdomyosarcomatous differentiation were negative for FKHR rearrangement.
\end{abstract}

\section{Introduction}

Rhabdomyosarcoma occurs much more commonly in children than in adults in genitourinary tract, in which more than $90 \%$ is embryonal, mostly botryoid type. Adult rhabdomyosarcoma is extremely rare [1-7]. Because of its rarity in adults, proper diagnosis and classification can be a challenge. To make this matter more complicated, rhabdomyoblastic differentiation is one of commonly seen heterologous elements in sarcomatoid carcinoma of urinary bladder [7]. Adult rhabdomyosarcoma of urinary bladder can have morphologic features overlapping with small cell carcinoma and poorly differentiated urothelial carcinoma with rhabdomyoblastic differentiation [7]. In this report, we reported three cases of primary rhabdomyosarcomatous tumor of the urinary bladder, two of them were diagnosed as carcinoma with extensive rhabdomyosarcomatous differentiation based on very minor carcinomatous elements identified and

\footnotetext{
*Correspondence: bingz@uphs.upenn.edu

Department of Pathology and Laboratory Medicine, Hospital of the University of Pennsylvania, Philadelphia, PA, USA
}

one as rhabdomyosarcoma without carcinomatous elements identified in the tissue examined. In addition, we compared the histologic and immunohistochemical features of these urinary bladder tumors with rhabdomyosarcomatous differentiation to that of well characterized bladder small cell carcinoma.

\section{Material and methods}

The database of the Department of Pathology and Laboratory Medicine of the University of Pennsylvania was searched for bladder rhabdomyosarcoma, carcinoma with rhabdomyosarcomatous differentiation and small cell carcinoma for the period 1987-2010. One case of rhabdomyosarcoma and two cases of poorly differentiated urothelial carcinoma with extensive rhabdomyosarcomatous differentiation were identified, and the medical charts were reviewed and reported. In addition, 8 cases of pure bladder small cell carcinoma and 5 cases of poorly differentiated urothelial carcinoma with areas of small cell carcinoma were retrieved for the study. All specimens were fixed in a 10\% neutral-buffered formalin solution and processed routinely. Histologic sections were 
reviewed and representative blocks of each case were selected for immunohistochemical study. This research was approved by the University of Pennsylvania Institutional Review Board.

\section{Immunohistochemistry}

Immunohistochemical stain was performed on formalinfixed, paraffin-embedded 4-um tissue sections with the avidin-biotin immunoperoxidase complex method (LSAB2 system, Dako Corporation, Carpinteria, Calif) with diaminobenzedine as the chromogen and hematoxylin as the nuclear counterstain. Information about the antibodies used was summarized in Table 1. Antigens retrieval was done by incubating the tissue sections in a Black and Decker Vegetable Steamer for 20 minutes in Target Retrieval Solution (Dako) preheated to $99^{\circ} \mathrm{C}$. The negative control was performed by substituting the primary antibody with nonimmune mouse or rabbit serum. Approximate positive controls were used.

\section{FISH}

FISH assay for translocation involving EWS and FHKR was performed on paraffin sections using Vysis breakapart probes (Vysis) according to the manufacture's procedure guidelines.

\section{Case histories and pathology}

Primary bladder tumors with rhabdomyosarcomatous differentiation: tumor with pure rhabdomyoblastic differentiation a.k.a rhabdomyosarcoma (case 1) versus tumor with minor carcinomatous element, a.k.a rhabdomyosarcomatous carcinoma (cases 2 and 3).

The clinical and histological features were summarized in table 2 and 3. All are adult patient (one male and two female). All presented with hematuria, two underwent cystectomy and the third one underwent transurethral resection of bladder tumor (TURBT) twice. The tumors were exophytic and measured $3.2 \mathrm{~cm}$ in case 1 and $25 \mathrm{~cm}$ in case 2 in the cystectomy specimens. In case 3 the second TURBT was performed in this institution and consisted of tumoral tissue in fragments in three parts measuring $5.5 \times 5.5 \times 1.0 \mathrm{~cm}, 2.5 \times 2.5 \times 0.5 \mathrm{~cm}$ and

Table 1 Antibodies

\begin{tabular}{llll}
\hline Antibody & Type & Manufacturer & Dilution \\
\hline Myogenin & $\begin{array}{l}\text { Mouse } \\
\text { monoclonal }\end{array}$ & Dako & $1: 200$ \\
\hline AE1/3 & $\begin{array}{l}\text { Mouse } \\
\text { monoclonal }\end{array}$ & $\begin{array}{l}\text { Novacastra } \\
\text { Laboratories }\end{array}$ & $1: 400$ \\
\hline Pan-CK & $\begin{array}{l}\text { Mouse } \\
\text { monoclonal }\end{array}$ & $\begin{array}{l}\text { Novacastra } \\
\text { Laboratories }\end{array}$ & $1: 50$ \\
\hline Chromogranin & Rabbit polyclonal & Zymed/Invitrogen & $1: 100$ \\
\hline Synaptophysin & Rabbit polyclonal & Zymed/Invitrogen & $1: 50$ \\
\hline
\end{tabular}

Table 2 Clinical features

\begin{tabular}{llll}
\hline Case No. & Age (years)/Gender & Clinical presentation & Treatment \\
\hline $\mathbf{1}$ & $61 /$ Female & Hematuria & Cystectomy \\
\hline $\mathbf{2}$ & $58 /$ Male & Hematuria & Cystectomy \\
\hline $\mathbf{3}$ & $57 /$ Female & Hematuria & TURBT \\
\hline
\end{tabular}

$3.0 \times 3.5 \times 0.7 \mathrm{~cm}$ in aggregates respectively, with largest fragments measuring $1.6 \mathrm{~cm}, 1.7 \mathrm{~cm}$ and $1.7 \mathrm{~cm}$ respectively. All three tumors showed areas of small round cell morphology with brisk mitosis and uniformly small nuclei with no prominent nucleoli (Figure 1A, B). The pure rhabdomyosarcoma (Case 1) showed no apparent rhabdomyoblasts, while case 2 showed extensive rhabdomyoblastic features and case 3 showed focal rhabdomyoblastic features (Figure 1D). In addition to prominent small round cell feature, all of the three cases exhibited scattered marked nuclear anaplasia (Figure 1A, B) and pleomorphism and patchy coagulative necrosis. No distinct alveolar pattern is seen. No carcinomatous element was identified in pure rhabdomyosarcoma in 25 sections of the $3.2 \mathrm{~cm}$ tumor, and the carcinomatous elements were very focal in other two cases (Figure 1C). In case 2, rare neoplastic epithelial cells were seen in pagetoid spread in the overlying urothelium in extensive sampling of this large tumor in one out of 46 sections. In case 3, very focal invasive urothelial carcinoma component was identified only in the initial TURBT specimen and not in the second TURBT specimens despite the fact that all of the tissue was submitted for histologic evaluation. Tumors in case 1 and 2 showed deep invasion into perivesicular tissue with lymph node metastasis.

Bladder tumors with small cell carcinoma component: Pure small cell carcinoma and poorly differentiated urothelial carcinoma with small cell carcinoma components.

There is no morphologic difference in the small cell carcinoma components between pure small cell carcinoma and poorly differentiated urothelial carcinoma with small cell carcinoma components. The tumors were composed of loosely arranged sheets and nests of small round blue cells with brisk mitosis and salt pepper chromotins (Figure 2B). Nuclear anaplasia was common. No rhabdomyoblastic differentiation is identified. The only difference was that areas of poorly differentiated conventional urothelial carcinoma were seen in the latter group.

\section{Immunohistochemical study}

Immunohistochemical studies with adequate controls were performed and the results were summarized in table 4. As shown in the table, the rhabdomyosarcomatous areas of all of three rhabdomyosarcomatous tumors of the bladder showed positivity for myogenin and 
Table 3 Histologic features

\begin{tabular}{lllllllll}
\hline Case & Size $\mathbf{( c m )}$ & $\begin{array}{l}\text { Carcinoma } \\
\text { Component }\end{array}$ & $\begin{array}{l}\text { Mitosis } \\
\text { (HPF) }\end{array}$ & Necrosis & Anaplasia & Rhabdomyoblasts & Depth of invasion & Lymph node Metastasis \\
\hline $\mathbf{1}$ & 3.2 & No & 17 & Yes & Yes & No & perivesicular tissue & $4 / 35$ \\
\hline $\mathbf{2}$ & 25 & Yes & 3 & Yes & Yes & Yes (diffuse) & PVT & $1 / 1$ \\
\hline $\mathbf{3}$ & 4 (ultrasound) & Yes (very focal) & 9 & Yes & Yes & Yes & At least detrusor muscle & N/A \\
\hline
\end{tabular}

desmin. Both of the pure rhabdomyosarcoma or rhabdomyosarcomatous carcinoma (carcinoma with extensive rhabdomyosarcomatous differentiation) showed similar extensive myogenin positivity (Figure 2E). No cytokeratin (Figure 2C) or chromogranin reactivity was detected in the rhabdomyosarcomatous areas of these tumors. Cytokeratin reactivity was only restricted to the rare pagetoid carcinomatous cells in case 2 and focal urothelial carcinoma in the case 3. Interestingly, Two out of three tumors (case 1 and 2) were also diffusely positive for synaptophysin (Figure 2G), but negative for chromogranin. Six out of 8 pure small cell carcinoma of the bladder and 3 out of 5 small cell carcinoma components in poorly differentiated urothelial carcinoma were evaluated for cytokeratin, chromogranin and synaptophysin reactivity. All cases tested were positive for cytokeratin (Figure 2D). All but 2 were positive for chromogranin. 7 cases of small cell carcinoma and 2 case of small cell carcinoma components of urothelial carcinoma were also stained for synaptophysin and all were positive
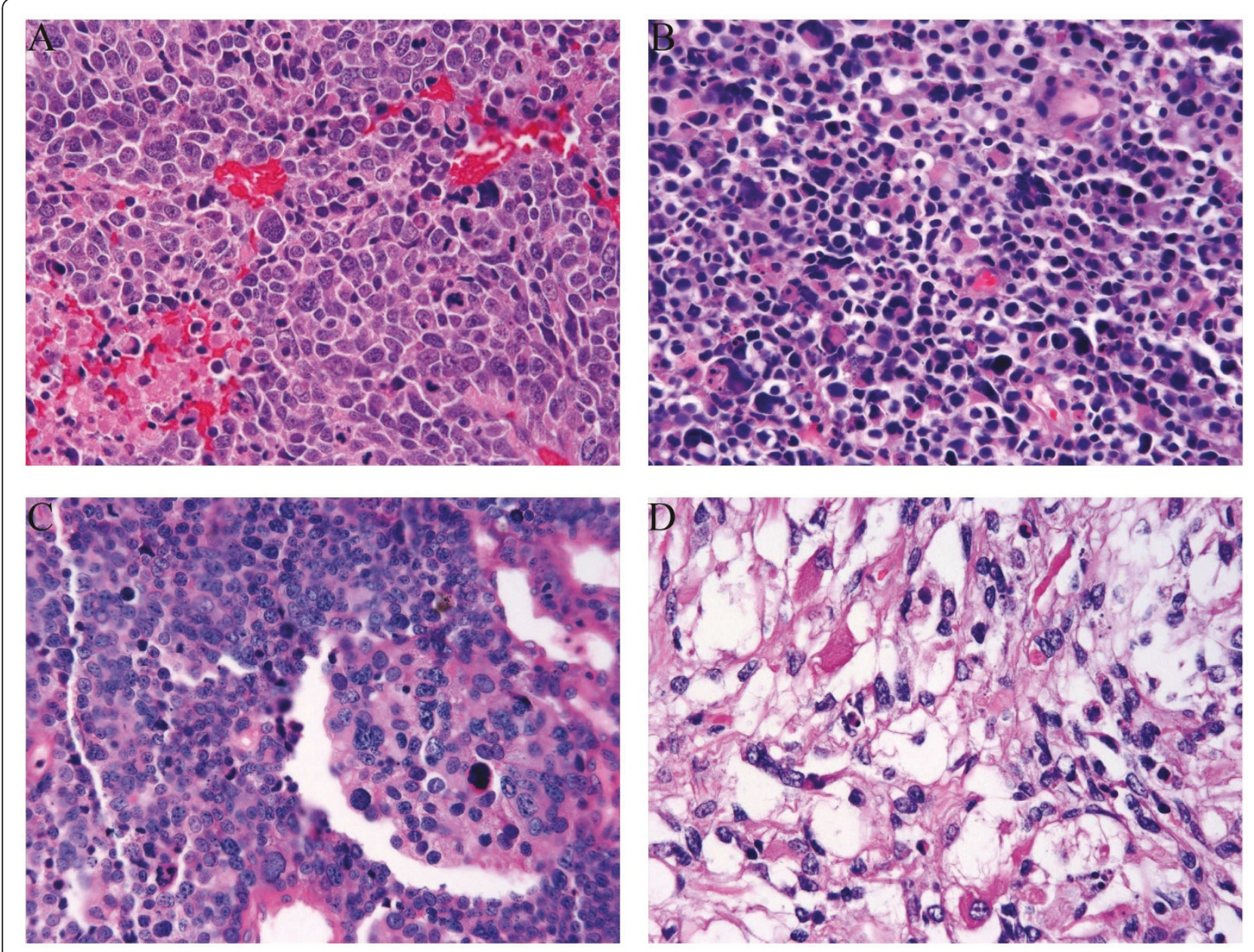

Figure 1 Morphology of primary bladder rhabdomyosarcoma and bladder tumor with extensive rhabdomyosarcomatous and focal epithelial differentiation, H\&E, 400x. A. Primary rhabdomyosarcoma with anaplasia. B, C, D. Bladder tumor with extensive

rhabdomyosarcomatous differentiation and focal epithelial differentiation showing anaplasia 400x (B), focal epithelial differentiation (C), rhabdomyoblastic differentiation (D). 

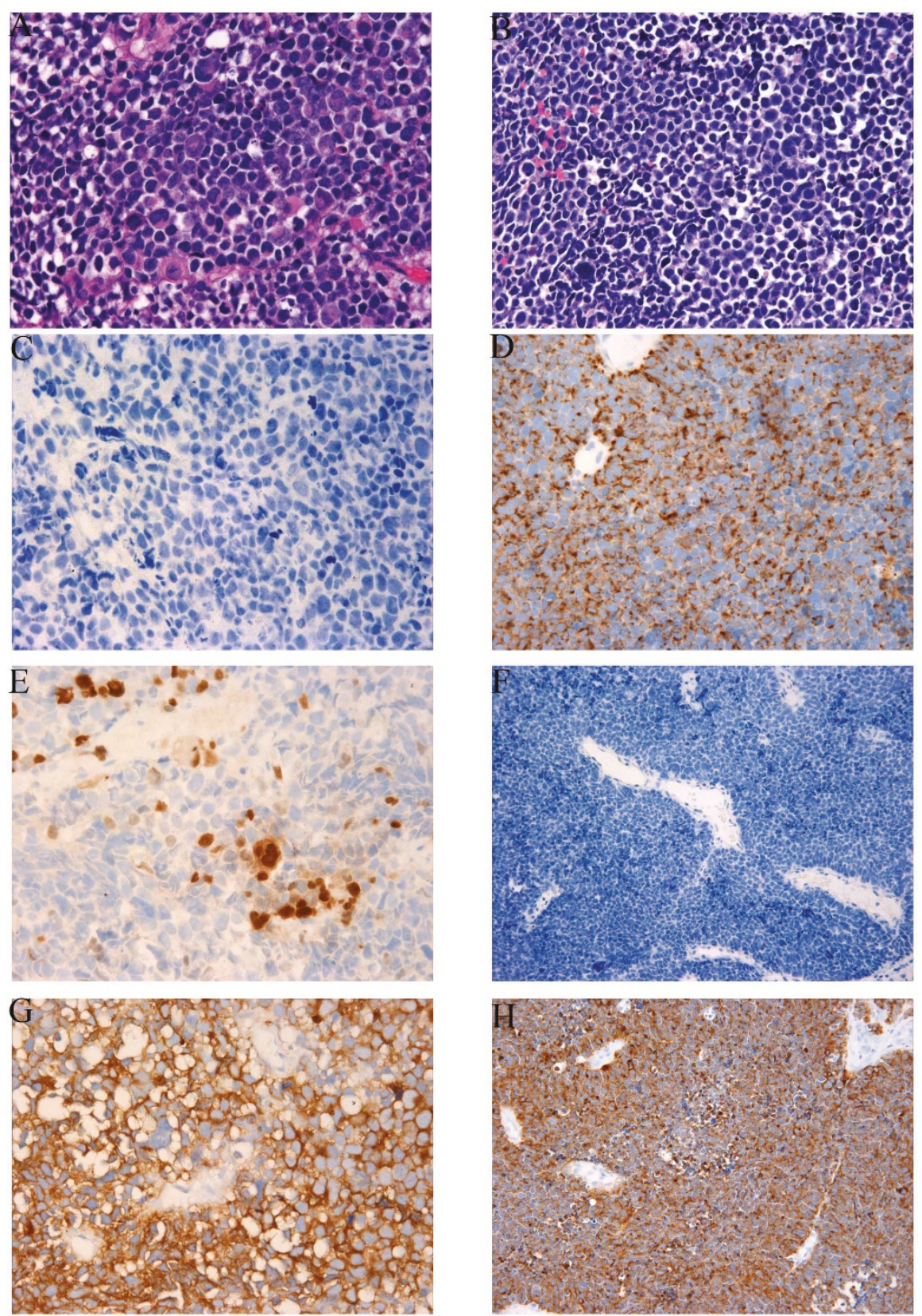

Figure 2 Immunohistochemical profiles of primary urinary bladder rhabdomyosarcoma and small cell carcinoma (400x). A, C, E, G, primary urinary bladder rhabdomyosarcoma. A, H\&E; C. Cytokeratin A/E1/3; E. Myogenin; G. Synaptophysin; B, D, F, H, small cell carcinoma of the urinary bladder. B. H\&E; D. Cytokeratin AE1/3; F. Myogenin; H. Synaptophysin.

(Figure 2H). All of 13 cases of the small cell carcinoma component from the pure small cell carcinoma and urothelial carcinoma with small cell carcinoma components were negative for myogenin (Figure 2F).

\section{FISH assays}

No EWS and FHKR breakapart signals were detected in any of three rhabdomyosarcomatous areas of the three bladder tumors.

\section{Discussion}

RMS in the urinary bladder has been well documented in children with the majority being embryonal, betryoid type $[8,9]$. RMS in adult urinary bladder is rare, with only scattered case reports or small series $[2,6,7,10-15]$. In the reported cases, the tumor usually occurs in older patients with the average age of $63+/-13$ years. There is a predilection for men with a male to female ration roughly $2: 1$. 
Table 4 Immunohistochemical study of Rhabdomyosarcomatous tumors and small cell carcinoma

\begin{tabular}{|c|c|c|c|c|c|c|}
\hline Cases & $\begin{array}{l}\text { Number of } \\
\text { cases }\end{array}$ & Myogenin & Cytokeratin & Synaptophysin & Chromogranin & $\begin{array}{l}\text { FISH in } \\
\text { RMS }\end{array}$ \\
\hline RMS* & 1 & Positive & Negative & Positive & Negative & negative \\
\hline RMS in $\mathrm{ca}^{\wedge}$ & 2 & $\begin{array}{l}\text { Positive in } \\
\text { RMS }\end{array}$ & $\begin{array}{l}\text { Negative in RMS; positive in } \\
\text { ca }\end{array}$ & $\begin{array}{l}\text { Positive in RMS in } 1 \text { out of } 2 \\
\text { cases }\end{array}$ & Negative & negative \\
\hline $\begin{array}{l}\text { Small cell } \\
\text { carcinoma }\end{array}$ & 13 & Negative & Positive (9/9) & Positive(9/9) & Positive in $7 / 9$ & N/A \\
\hline
\end{tabular}

FISH DNA breakapart signal analysis for detection of translocation associated with EWS and FHKR.

*RMS: Rhabdomyosarcoma or rhabdomyosarcomatous component in appropriate setting; ^ca: Carcinoma.

RMS in adult is composed of small round blue cells with high nuclear cytoplasm ratio, brisk mitosis and frequent apoptosis. Frequently the tumors show nuclear anaplasia, with random large anaplastic cells scattered in the tumor, similar to the nuclear anaplasia seen in the Wilm's tumor without the requirement of tripolar atypical mitosis [16]. RMS in adult urinary bladders has been reported to be alveolar [7,14,17], embryonal [11-13,17], pleomorphic $[2,6]$ type or unspecified [10].

Rhabdomyoblastic morphology may not be present in the tumor; therefore it may be confused with other primitive tumors, especially with small cell carcinoma. On the other hand, variable aberrant rhabdomyoblastic differentiation could be seen in poorly differentiated urothelial carcinoma. Typically, differential diagnosis of RMS in adults includes sarcomatoid urothelial carcinoma with extensive rhabdomyosarcomatous differentiation and other tumors with small round cell morphology including small cell carcinoma, plasmacytoid urothelial carcinoma, primitive neuroectodermal tumor and lymphoma. Immunohistochemical analysis has an important role in the differential diagnosis of these tumors. Desmin and myogenin immunoreactivity can be used to differentiate rhabdomyosarcoma from other round cell tumors without rhabdomyoblastic differentiation but not sarcomatoid carcinoma with rhabdomyoblastic differentiation. In practice, urothelial carcinoma with extensive rhabdomyosarcomatous differentiation can only be differentiated from de novo rhabdomyosarcoma based on finding of any carcinomatous (neoplastic epithelial) element and/or unequivocal cytokeratin immunoreactivity in tumor cells. Therefore, sufficient sampling and mapping of tumor to look for any carcinomatous element, in-situ or invasive, are critical for making the distinction between these two entities. The diagnostic difficulties are reflected in two of our cases. In case 2 , the tumor is very large $(25 \mathrm{~cm})$ and only very small focus showing carcinomatous differentiation. In case 3 , small focus of urothelial carcinoma was only identified in the initial TURBT specimen. In the subsequent TURBT, no carcinomatous component was identified albeit the whole specimen was entirely submitted in 15 cassettes for histologic evaluation. One can conceive that when the carcinomatous element is very small and/or dominated or replaced by rhabdomyosarcomatous element, the minor carcinomatous element might not be morphologically detectable easily in routine sampling. In addition, as seen in these two cases, the rhabdomyoblastic element could also lose their other epithelial characteristics such as cytokeratin reactivity. In such case, diagnosis of de novo rhabdomyosarcoma is basically a function of extensiveness of tissue sampling except for alveolar rhabdomyosarcoma for which molecular analysis for PAX3/7-FHKR translocation can be used to confirm the diagnosis. As illustrated in our cases, majority of the rhabdomyoblastic tumors of the bladder are likely rhabdomyosarcomatous carcinoma as small foci of carcinomatous element are only detectable through extensive sampling of the single or multiple specimens and pure rhabdomyoblastic tumor (a.k.a de novo rhabdomyosarcoma) is very rare and the diagnosis should be made with extreme caution.

The other challenge is differentiating rhabdomyosarcoma and rhabdomyoblastic carcinoma from small cell carcinoma. As some previous studies showed [7], we also find that there is significant overlapping of morphologic features between small cell carcinoma, rhabdomyosarcoma and carcinoma with extensive rhabdomyoblastic differentiation in urinary bladder. Small cell carcinoma of bladder is a rare tumor with incidence reported between $0.5 \%$ and $1 \%$ of the bladder cancers in retrospective studies [18-20]. It can coexist with urothelial carcinoma. It has been shown that similar patterns of allelic loss in the small cell carcinoma component coexisting with urothelial carcinoma. The similar patterns of allelic loss leads to the hypothesis that these cells have common clonal origin [21]. The prognosis for small cell carcinoma of the urinary bladder is poor, with overall 1 -year and 5-year disease-specific survival rates of $56 \%$ and $16 \%$ respectively [19]. The treatment for bladder small cell carcinoma is not standardized. However, chemotherapy played a prominent role in the management of these tumors [22]. Recent development includes the use of adjuvant platinum-based chemotherapy for advanced disease. For adult bladder RMS, treatments are variable, including surgical resection, radiotherapy, chemotherapy or combined therapy. Prognostic markers with a worse 
prognosis included nonembryonal histology, tumor invasion and tumor size $>5 \mathrm{~cm}$ in children' RMS [23]. Management of carcinoma with extensive rhabdomyosarcomatous differentiation is not well defined. The preferred treatment appears to be cystectomy followed by radiation therapy or chemotherapy [24].

Due to the difference in management, proper classification of the rhabdomyoblastic tumors in bladder and differentiate these tumors from morphologic mimics such as small cell carcinoma are important. In this study, we compared the expression of cytokeratin, myogenin, synaptophysin and chromogranin in rhabdomyosarcomatous tumor and small cell carcinoma of the bladder. As shown in Table 4, areas of rhabdomyosarcomatous differentiation were positive for desmin and myogenin, negative for cytokeratin and chromogranin in all cases. In this study, we reported strong positivity for synaptophysin detected in the RMS component of one of the sarcomatoid carcinomas, which was not reported previously. As reported [7], positivity for synaptophysin can also be detected in pure RMS, and hence has little utility to differentiate rhabdomyosarcomatous tumors from other mimics with neuroendocrine differentiation such as small cell carcinoma. In contrast, small cell carcinoma components, whether it is pure or admixed with urothelial carcinoma, were positive for cytokeratin and largely positive for chromogranin, and negative for myogenin. FISH analysis was performed on paraffin-embedded formalin-fixed tissue in three cases of rhabdomyosarcomatous tumor of the bladder tumors to detect rearrangement of the FKHR (13q14) region which is hallmark of alveolar rhabdomyosarcoma. No FKHR rearrangement was detected in any of these three cases. The negativity for such arrangements does not support the diagnosis of alveolar type of rhabdomyosarcoma. Primary de novo rhabdomyosarcoma of any type is very rare in adult bladder. In this setting, only alveolar rhabdomyosarcoma can be reliably diagnosed by molecular test for PAX3 or 7-FKHR translocation. If the tumor is negative for PAX3 or 7-FKHR translocation and lack of morphologic pleomorphism, most of rhabdomyosarcomatous tumors arising from adult bladder are likely of rhabdomyosarcomatous carcinoma, a form of sarcomatoid carcinoma. Embryonal rhabdomyosarcomas can arise in bladder wall in pediatric patient but are extremely rare in adult patient. Clear morphologic differential separation of de novo embryonal rhabdomyosarcoma from those rhabdomyosarcomatous carcinomas would be a challenge and depend upon one's will to search for evidence of carcinoma in specimen. Unless a molecular test reveals evidence of alveolar rhabdomyosarcoma, tumors with extensive rhabdomyosarcomatous differentiation of the adult bladder should be either classified as rhabdomyosarcomatous sarcomatoid carcinoma when epithelial differentiation is identified or rhabdomyosarcomatous tumor of the bladder when no epithelial differentiation can be detected after reasonable effort to search such evidence in a caveat that there is no standard for what is considered reasonable effort in evaluating such specimen. Such diagnostic approach acknowledges the difficulty in making morphologic differentiation between rare occurring de novo soft tissue rhabdomyosarcoma and rhabdomyosarcomatous sarcomatoid carcinoma in adult patients.

In summary, pure RMS is very rare in adult urinary bladder while rhabdomyosarcomatous sarcomatoid carcinoma is more common. Adequate sampling, search for admixed in-situ or invasive urothelial carcinoma and use of cytokeratin stain may help to reach a correct diagnosis. A descriptive diagnosis of rhabdomyosarcomatous tumor of the bladder is preferred in difficult cases. Rhabdomyosarcoma and rhabdomyosarcomatous sarcomatoid carcinoma can be differentiated from small cell carcinoma of the bladder by immunohistochemical evaluation of cytokeratin and chromogranin and myogenin.

Authors' contributions

ZB and PJZ conceived, designed and carried out the project. All authors read and approved the final manuscript.

\section{Competing interests}

The authors declare that they have no competing interests.

Received: 24 May 2011 Accepted: 15 July 2011 Published: 15 July 2011

\section{References}

1. al-Jaberi TM, al-Masri N, Tbukhi A: Adult rhabdomyosarcoma of the gall bladder: case report and review of published works. Gut 1994, 35(6):854-856.

2. Aydoganli L, Tarhan F, Atan A, Akalin Z, Yildiz M: Rhabdomyosarcoma of the urinary bladder in an adult. Int Urol Nephrol 1993, 25(2):159-161.

3. Childs L, Hull D, Bostwick DG: Adult urinary bladder rhabdomyosarcoma. Urology 2008, 72(4):948 e941-943.

4. Evans AT, Bell TE: Rhabdomyosarcoma of the bladder in adult patients: report of three cases. J Urol 1965, 94(5):573-575.

5. Grigoriou I, Skrepetis K: Botryoid rhabdomyosarcoma of the bladder in an adult female. J Buon 2002, 7(4):385-387.

6. Lauro S, Lalle M, Scucchi L, Vecchione A: Rhabdomyosarcoma of the urinary bladder in an elderly patient. Anticancer Res 1995, 15(2):627-629.

7. Paner GP, McKenney JK, Epstein Jl, Amin MB: Rhabdomyosarcoma of the urinary bladder in adults: predilection for alveolar morphology with anaplasia and significant morphologic overlap with small cell carcinoma. Am J Surg Pathol 2008, 32(7):1022-1028.

8. Newton WA Jr, Soule EH, Hamoudi AB, Reiman HM, Shimada $\mathrm{H}$, Beltangady M, Maurer H: Histopathology of childhood sarcomas, Intergroup Rhabdomyosarcoma Studies I and II: clinicopathologic correlation. J Clin Oncol 1988, 6(1):67-75.

9. Pappo AS: Rhabdomyosarcoma and other soft tissue sarcomas in children. Curr Opin Oncol 1996, 8(4):311-316.

10. Ahlering TE, Weintraub P, Skinner DG: Management of adult sarcomas of the bladder and prostate. J Urol 1988, 140(6):1397-1399.

11. Kerr KM, Grigor KM, Tolley DA: Rhabdomyosarcoma of the adult urinary bladder after radiotherapy for carcinoma. Clin Oncol (R Coll Radiol) 1989, 1(2):115-116

12. Kunze $E$, Theuring F, Kruger G: Primary mesenchymal tumors of the urinary bladder. A histological and immunohistochemical study of 30 cases. Pathol Res Pract 1994, 190(4):311-332. 
13. Taylor RE, Busuttil A: Case report: adult rhabdomyosarcoma of bladder, complete response to radiation therapy. J Urol 1989, 142(5):1321-1322.

14. Zarabi CM, Huntrakoon M, Fine KD: Disseminated rhabdomyosarcoma of the urinary bladder in an adult. South Med J 1987, 80(4):526-529.

15. Ziari M, Sonparde G, Shen S, Zhai J, Teh BS, Lerner SP: Patients with unusual bladder malignancies and a rare cause of splenomegaly. Case 2. Rhabdomyosarcoma of the urinary bladder in an adult. J Clin Oncol 2005, 23(19):4459-4460.

16. Vujanic GM, Sandstedt B: The pathology of Wilms' tumour (nephroblastoma): the International Society of Paediatric Oncology approach. J Clin Pathol 2010, 63(2):102-109.

17. Lambert I, Debiec-Rychter M, Dubin M, Sciot R: Solid alveolar rhabdomyosarcoma originating from the urinary bladder in an adult. Diagnostic value of molecular genetics. Histopathology 2004, 44(5):508-510.

18. Abrahams NA, Moran C, Reyes AO, Siefker-Radtke A, Ayala AG: Small cell carcinoma of the bladder: a contemporary clinicopathological study of 51 cases. Histopathology 2005, 46(1):57-63.

19. Cheng L, Pan CX, Yang XJ, Lopez-Beltran A, MacLennan GT, Lin H, Kuzel TM, Papavero V, Tretiakova M, Nigro K, et al: Small cell carcinoma of the urinary bladder: a clinicopathologic analysis of 64 patients. Cancer 2004, 101(5):957-962.

20. Pant-Purohit M, Lopez-Beltran A, Montironi R, MacLennan GT, Cheng L: Small cell carcinoma of the urinary bladder. Histol Histopathol 25(2):217-221

21. Higashiyama M, Doi O, Kodama K, Yokouchi H, Tateishi R: High prevalence of bcl-2 oncoprotein expression in small cell lung cancer. Anticancer Res 1995, 15(2):503-505.

22. Ismaili N, Heudel PE, Elkarak F, Kaikani W, Bajard A, Ismaili M, Errihani $H_{\text {, }}$ Droz JP, Flechon A: Outcome of recurrent and metastatic small cell carcinoma of the bladder. BMC Urol 2009, 9:4.

23. Rodeberg DA, Anderson JR, Arndt CA, Ferrer FA, Raney RB, Jenney ME, Brecht IB, Koscielniak E, Carli M, Bisogno G, et al: Comparison of outcomes based on treatment algorithms for rhabdomyosarcoma of the bladder/ prostate: combined results from the Children's Oncology Group, German Cooperative Soft Tissue Sarcoma Study, Italian Cooperative Group, and International Society of Pediatric Oncology Malignant Mesenchymal Tumors Committee. Int J Cancer 128(5):1232-1239.

24. Stamatiou K, Galariotis N, Michailidis I, Petrakopoulou N, Moustou H, ZiziSermpetzoglou A: Sarcomatoid carcinoma of the urinary bladder: a clinicopathological study of 4 cases and a review of the literature. Korean J Urol 51(10):724-728.

doi:10.1186/1746-1596-6-66

Cite this article as: Bing and Zhang: Adult urinary bladder tumors with rabdomyosarcomatous differentiation: Clinical, pathological and immunohistochemical studies. Diagnostic Pathology 2011 6:66.

\section{Submit your next manuscript to BioMed Central and take full advantage of:}

- Convenient online submission

- Thorough peer review

- No space constraints or color figure charges

- Immediate publication on acceptance

- Inclusion in PubMed, CAS, Scopus and Google Scholar

- Research which is freely available for redistribution

Submit your manuscript at www.biomedcentral.com/submit
Biomed Central 